\title{
Discrimination of empty duration in the click sequence simulating a mora structure
}

\author{
Masako Tanaka, Minoru Tsuzaki, and Hiroaki Kato \\ ATR Human Information Processing Research Laboratories, \\ 2-2 Hikaridai, Seika-cho, Soraku-gun, Kyoto, 619-02 Japan \\ (Received 18 June 1993)
}

Keywords: Temporal structure, Discrimination threshold, Click sequence PACS number: 43. 66. $\mathrm{Mk}$

\section{Introduction}

The relation between physical duration and subjective duration has been studied psychophysically for separately presented time intervals (single patterns) to understand the mechanism of time perception in hearing research. It seems, however, that it is more common to perceive successively presented intervals than separately presented intervals.

Speech and music, for example, consist of successive intervals. Although speech and music have different physical characteristics, we can relate singing and hand clapping with musical rhythm. This suggests the possibility of extracting the temporal structure from each different stimulus and comparing all of them in terms of a common mental representation. We can hypothesize the existence of such a common mental representation by finding common rules for general perception of time intervals regardless of the stimuli of speech or non-speech.

Hirsh, Monahan, and Singh ${ }^{1)}$ studied the human's ability to detect the timing deviation of a single element in a periodic series of tones. Using click sequences, they reported that the detectability of an irregularity in a interval was higher than that in an initial interval for a standard interval of $50 \mathrm{~ms}$. Kato, Tsuzaki, and Sagisaka, ${ }^{2)}$ on the other hand, studied discrimination of the segmental duration in words; the length of a single mora in Japanese words was modified. They reported a smaller discrimination threshold for an initial mora position than for a final mora position in words. Although these two studies apparently contradict one another, there were differences in the types of employed stimuli such as speech or click sequences, and differences in the procedures to detect irregularities in each sequence and to discriminate the two sequences.

In this study, we measure the discrimination threshold for click sequences by a method and procedure similar to those of Kato et al. to investigate whether the difference in stimulus type causes the different results. We also investigate the effects caused by the factors of homogeneity and base words, in addition to the position of the element modified in the sequence.

\section{Method}

A standard stimulus consisted of three consecutive empty intervals $(t 1, t 2, t 3)$ divided by four clicks. Table
1 shows the physical duration of these intervals for four types of standard stimuli. $\mathrm{A}(\mathrm{Het})$ and $\mathrm{B}(\mathrm{Het})$ each consisted of a sequence simulating the temporal structure of Japanese word, "SHINAGIRE" or "NAMERAKA." These two words were used in experiments by Kato et al. ${ }^{2}$ ) The clicks (duration markers) for the starting points of individual moras were located at the power-dip close to the starting points of individual consonants. $\mathbf{A}(\mathrm{Hom})$ and $\mathbf{B}(\mathrm{Hom})$ were sequences comprised of the average duration for the three intervals of $\mathrm{A}(\mathrm{Het})$ and $\mathrm{B}(\mathrm{Het})$, respectively.

The comparison stimulus had the same sequence as the standard stimulus, and the physical duration of the first empty interval $(t 1)$ or the third empty interval $(t 3)$ was modified from $-60 \mathrm{~ms}$ to $+60 \mathrm{~ms}$ in $2.5 \mathrm{~ms}$ steps for each standard sequence. The duration markers had a single rectangular waveform of $1,000 \mathrm{~Hz}$, and had a duration of $1 \mathrm{~ms}$. These markers were presented diotically through headphones (STAX SR- $\Lambda$ Pro). Each of them had a sound level measured by an artificial ear (Brüel \& Kjaer 4153), of about 78 dBA.

The subjects (seven females all with normal hearing) were required to respond whether the two stimuli were "same" or "different." The up-down method was used to measure the discrimination threshold.

\section{Results}

The discrimination thresholds were calculated by averaging the upward threshold and the downward threshold. A repeated measures analysis of variance (ANOVA) ( 2 modified positions $\times 2$ base words $\times 2$ homogeneity/heterogeneity factors), with the subjects as the blocking factor for these discrimination thresholds were performed. The significant main effects were the modified position $[F(1,42)=36.48, p<0.001]$, and the homogeneity $[F(1,42)=7.13, p<0.010]$. No significant interaction of these factors was observed. Figure 1 shows discrimination thresholds; they are averaged for the seven subjects.

\section{Discussion}

The result concerning the effect of the modified position, i.e. the discrimination threshold for $t 1$ is smaller than that for $t 3$ in a click sequence, agrees with the experimental results obtained by Kato et al. ${ }^{2)}$ This suggests the possibility that a common mechanism 
Table 1 Physical Duration of Three Intervals.

\begin{tabular}{lrcc}
\hline \multirow{2}{*}{$\begin{array}{c}\text { Stimulus } \\
\text { condition }\end{array}$} & \multicolumn{3}{c}{ Temporal position } \\
\cline { 2 - 4 } & $t 1$ & $t 2$ & $t 3$ \\
\hline A (Het) & 185 & 160 & 175 \\
A (Hom) & 173 & 173 & 173 \\
B (Het) & 155 & 182 & 163 \\
B (Hom) & 166 & 166 & 166 \\
\hline
\end{tabular}

$\mathrm{A}(\mathrm{Het})$ : Sequence simulating the temporal structure of "SHINAGIRE"

$\mathrm{A}(\mathrm{Hom})$ : Homogeneous sequence comprised of the average duration of $\mathrm{A}(\mathrm{Het})$

$\mathrm{B}(\mathrm{Het})$ : Sequence simulating the temporal structure of "NAMERAKA"

$\mathrm{B}(\mathrm{Hom})$ : Homogeneous sequence comprised of the average duration of $\mathrm{B}(\mathrm{Het})$

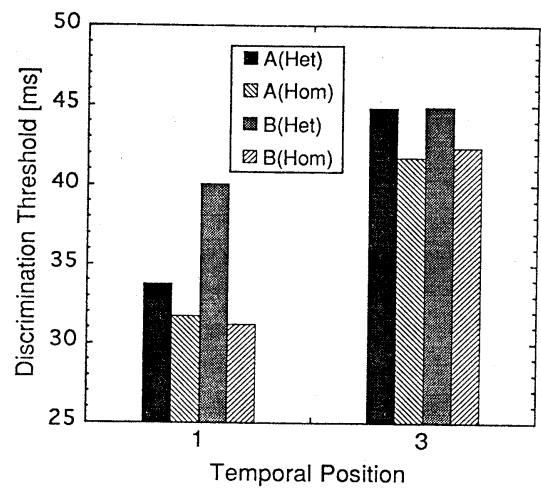

Fig. 1 Discrimination threshold for temporal interval of click sequence as a function of the temporal position, homogeneity, and base words.

works to perceive the timing structure of speech and click sequences. The results of Hirsh et al., ${ }^{1)}$ however, indicated no significant effect by the position for a standard interval of $200 \mathrm{~ms}$, which approximately corresponds to the temporal interval used in our experiment. This difference might be caused by the different procedures used in the two experiments; Hirsh et al. investigated the human's ability to detect irregularities in temporal patterns and we investigated the human's ability to discriminate two temporal patterns.

Adding to the positional effect, the discrimination thresholds for sequences simulating the temporal structure of words were larger than those for homogeneous sequences. The physical variance in the heterogeneous condition is able to increase the variance of a mental representation and increase the threshold. The effect of homogeneity suggests the discrimination process was affected by the other intervals surrounding the target interval in the sequence, although the physical difference in the two temporal patterns could only be found in the target interval. Assuming that the degree of influence by the other intervals was more for $t 3$ than $t 1$, we could also explain the results that the discrimination thresholds for $t 3$ were larger than those for $t 1$.

For further studies, we plan to systematically investigate the effects of position and homogeneity for an increased number of intervals.

\section{Conclusion}

On the discrimination of temporal structures in two sequences, we found that 1) the discrimination for the first interval is more sensitive than that for the third interval in a sequence, and that 2) the discrimination thresholds for heterogeneous conditions are larger than those for homogeneous conditions.

\section{References}

1) I. J. Hirsh, C. B. Monahan, K. W. Grant, and P. G. Singh, "Studies in auditory timing: 1 . Simple patterns," Percept. Psychophys. 47, 215-226(1990).

2) H. Kato, M. Tsuzaki, and Y. Sagisaka, "Discrimination threshold for segmental duration in words-Effects of mora position, $F_{0}$ contour, and vowel color-," Tech. Rept. Hear. Acoust. Soc. Jpn., H-91-43 (1991) (in Japanese).

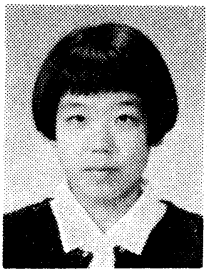

Masako Tanaka was born in 1968. She received the B.M. degree in Musicology from Osaka University of Arts, Osaka, in 1990, and the M.E. degree in acoustics from Kyushu Institute of Design in Fukuoka, in 1992. Since 1992, she has been working in the ATR Human Information Processing Research Laboratories, Kyoto, Japan, as a research associate.

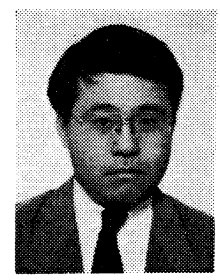

Minoru Tsuzaki was born in 1957. He received the B.A. and M.A. degrees in psychology from the University of Tokyo, Tokyo, in 1980 and 1982. He worked as an instructor in experimental psychology at Niigata University, Japan, from 1982 to 1985 , and at University of Tokyo from 1985 to 1988 . He worked in the ATR Auditory and Visual Perception Research Laboratories, Kyoto, Japan, from 1988 to 1992. Since 1992, he has been with the ATR Human Information Processing Research Laboratories, where he is currently a senior researcher.

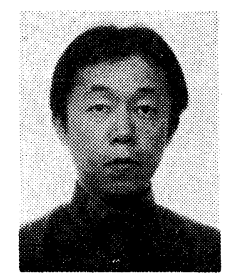

Hiroaki Kato was born in 1961. $\mathrm{He}$ receives the B.E. and M.E. degrees in instrumentation engineering from Kobe University, Kobe, in 1986 and 1988. He worked in the ATR Auditory and Visual Perception Research Laboratories, Kyoto, Japan, from 1988 to 1993 . Since 1992 . He has been with the ATR Human Information Processing Research Laboratories as a researcher. 